\title{
Sozialen Nutzen belegen
}

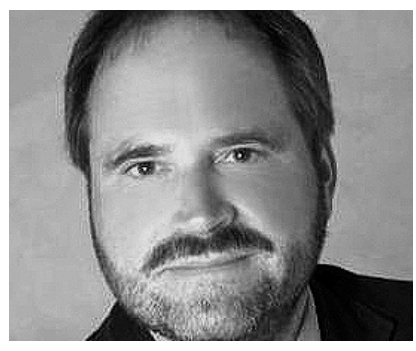

VON KLAUS-ULRICH SCHELLBERG Prof. Dr. Klaus-Ulrich Schellberg ist Hochschullehrer für Betriebswirtschaftslehre von Sozialunternehmen und Studiengangsleiter für den Studiengang Sozialwirtschaft an der Evangelischen Hochschule Nürnberg. Er ist Mitgesellschafter von xit $\mathrm{GmbH}$ forschen.planen.beraten in Nürnberg.

Internet

http://www.xit-online.de
Die Sozialwirtschaft steht immer wieder als Kostenfaktor am Pranger. Eine bedeutsame Aufgabe für die Zukunft ist es, dass soziale Verbände und Unternehmen lernen, die von ihnen produzierte Wertschöpfung nachweisen zu können. Mit dem Konzept des Social Return on Investment kann dieser "Mehrwert des Sozialen « auch im Einzelfall errechnet werden.

Seit dem ersten Auftauchen vor rund zehn Jahren hat sich der Begriff der Wertschöpfung in der Sozialwirtschaft schnell verbreitet. "Zukunft: Wertschöpfung durch Wertschätzung «, »In Soziales investieren - mehr Werte schaffen «; » Mehrwert des Sozialen Gewinn für die Gesellschaft «; »Selbstgesetzter Produktionsauftrag: Gesellschaftlichen Mehrwert erzeugen ", so lauteten etwa vergangene Titel der ConSozial und des Kongresses der Sozialwirtschaft. Durch seine vielfältige Verwendung droht der Begriff inflationär und unscharf zu werden. Es ist daher Zeit für den Versuch einer begrifflichen und konzeptionellen Präzisierung.

Im heutigen Verständnis beschreibt Wertschöpfung grundsätzlich die Wertänderung von Ressourcen durch einen Produktions- oder Transformationsprozess. Die Wertschöpfung ist demnach grundsätzlich die Differenz zwischen dem Wert der eingesetzten Ressourcen und dem Wert der geschaffenen Produkte oder Leistungen. Wertschöpfung entsteht demnach immer, wenn aus einem Ausgangszustand ein besserer, »wertvollerer " Endzustand erreicht wird. Wertschöpfung ist also der durch die wirtschaftliche Tätigkeit einer Wirtschaftseinheit, beispielsweise einem Unternehmen, geschaffene Mehrwert.

Wie die Bewertung erfolgt, ob in Geld oder in anderen Kategorien, ist dabei unerheblich. Der Begriff der Wertschöpfung kann unabhängig von Marktformen verwendet werden und er bezieht auch Dienstleistungen mit ein. Hierin unterscheidet sich der moderne Wertschöpfungsbegriff etwa vom klassischen und marxistischen Wertschöpfungsbegriff, in dem Wertschöpfung nur durch menschliche Arbeit erfolgte.

In einer allgemeinen Darstellung ist »Wertschöpfung « noch ein abstrakter Begriff (vgl. Abbildung S. 21). Es gelingt in der
Regel leicht, den Wert der eingesetzten Ressourcen in Geld zu bewerten (den Kaufpreise, Vergütungen). Der Wert der Leistung auf der Abnehmerseite ist jedoch nicht immer in Geld zu bewerten, häufig wurde er auch niemals wirklich bewusst »errechnet«. Wer könnte etwa in Geldeinheiten den Wert eines romantischen Abendessens in einem schönen Restaurant bemessen? Der Nutzen des Kunden wird mindestens so hoch sein wie der Betrag, der hierfür im Restaurant bezahlt wird - sonst würde wohl niemand freiwillig dorthin gehen. Dies wird in der Ökonomie mit der Theorie der bekundeten Präferenz bezeichnet. Instrumente, den Nutzen weitergehend oder gar objektiv zu bestimmen, sind allerdings rar.

Der Wert, für den Güter und Leistungen getauscht werden, bestimmt den Transaktionswert (in der Regel Marktpreise). Hierdurch wird die Verteilung der Wertschöpfung bestimmt (vgl. Abb. 2). Ein Teil der Wertschöpfung verbleibt beim Unternehmen, dies ist die unternehmensbezogene Wertschöpfung (»Company Value Added «). Ein Teil der Wertschöpfung erhält der Kunde als »Mehrwert « über dem Marktpreis. Dies ist die kundenbezogene Wertschöpfung (»Customer Value Added «).

Die Verteilung der Wertschöpfungsgewinne auf Kunden oder Unternehmen ist eine Frage der Austauschbeziehungen und der Marktverhältnisse. Funktioniert beispielsweise der Markt und es herrscht starker Wettbewerb, so werden die Preise unter Druck kommen und der Transaktionswert wird nicht weit über dem Wert der eingesetzten Ressourcen sein. Die kundenbezogene Wertschöpfung ist dann höher. Die Wertschöpfung wird im Rahmen der volkswirtschaftlichen Gesamtrechnung statistisch erhoben.

In der volkswirtschaftlichen Gesamtrechnung ist die Bruttowertschöpfung eine zentrale Messgröße. Sie ist definiert als der Wert aller erbrachten Güter und Dienstleistungen einer Volkswirtschaft oder eines Wirtschaftszweiges abzüglich der eingesetzten Vorleistungen (also bezogene Sachund Dienstleistungen (vgl. Abb. 3).

Die Sozialwirtschaft wird nicht als eigener Wirtschaftszweig ausgewiesen, sondern 
die einzelnen Arbeitsfelder sind einzelne Positionen der Rubrik »öffentliche und soziale Dienstleister «. Ihr Volumen wird mit 152 Milliarden Euro aus (Bundesarbeitsgemeinschaft der Freien Wohlfahrtspflege in der Presseerklärung zum 6. Kongress der Sozialwirtschaft 2009) geschätzt. In einer statistischen Analyse für Bayern wurden 6,9 Prozent der Bruttowertschöpfung der Gesundheits- und Sozialbranche zugerechnet (Puch/Schellberg, Vortrag auf der ConSozial 11.11.2009); hochgerechnet auf das Bundesgebiet sind dies circa 140 Milliarden Euro.

\section{Grenzen der volkswirtschaftlichen Gesamtrechnung für die Sozialwirtschaft}

Die Wertschöpfung in der volkswirtschaftlichen Gesamtrechnung errechnet sich aus der Differenz des Umsatzes (des Transaktionswertes) und der bezogenen Vorleistungen aus anderen Wirtschaftsbe- reichen (Teil der eingesetzten Ressourcen). Die Vergütung der Mitarbeitenden und der eingesetzten Investitionen ist Teil der Wertschöpfung. Die volkswirtschaftliche Gesamtrechnung wählt als Bezugspunkt die unternehmensbezogene Wertschöpfung. Den zusätzlichen Nutzen, die kundenbezogene Wertschöpfung, erfasst sie über die Wertschöpfung nachgelagerter Wirtschaftszweige (also etwa, wenn die Schraubenhersteller ihre Produkte billig verkaufen, steigt die Wertschöpfung beim KfZ-Hersteller). Der zusätzliche Nutzen beim Endverbraucher am Ende der Wertschöpfungskette wird nicht betrachtet.

An dieser Stelle setzt die Diskussion über Wertschöpfung in der Sozialwirtschaft an. Die Sozialwirtschaft steht immer wieder unter dem Argumentationsdruck, sie sei nur Kostenfaktor, produziere also keine Wertschöpfung. Das Gegenargument lautet, der Wert der in der Sozialwirtschaft erbrachten Leistungen sei höher als die hierfür von der öffentlichen Hand oder der
Gesellschaft aufgebrachten Mittel (dem Transaktionswert). Dies könnte dann als der »Mehrwert des Sozialen « bezeichnet werden.

Die Sozialwirtschaft stößt damit in ein Problem der Ökonomie vor: Der "wahre " Nutzen von Leistungen wird von der Ökonomie nicht betrachtet, da ja der Kunde als autonomer, mündiger Entscheider betrachtet wird, dem in einem freiheitlichen System keine Vorgaben gemacht werden (sollen). Dementsprechend hat die Ökonomie auch kaum Analyse- oder Entscheidungsinstrumente für eine objektive Bewertung von Kundenentscheidungen entwickelt. Genau dies benötigt jedoch die Sozialwirtschaft.

\section{Social Return on Investment als Lösung für das Bewertungsproblem}

In den letzten Jahrzehnten wurden verschiedene Formen von Kosten-NutzenAnalysen für öffentliche Vorhaben durch-

\section{Fünf SROIs für verschiedene Abnehmersysteme}

Ein Social Return on Investment für Sozialunternehmen muss zwei Probleme lösen: Zum Ersten muss er die Abnehmer des Sozialunternehmens identifizieren (Leistungsempfänger, öffentliche Träger, Gesellschaft etc.) und dann zum Zweiten eine Bewertung für deren Nutzen finden.

Es ist nach momentanem Erkenntnisstand nicht möglich, einen einheitlichen Nutzen für die verschiedenen Abnehmer eines Sozialunternehmens zu finden. Die soziale Wirkung beim Leistungsempfänger ist eine andere als die bei der öffentlichen Hand. Die Wirkung für die Gesellschaft, unter Berücksichtigung des Sozialsystems, ist eine andere als für die Wirtschaft. Andererseits wird es auch manche Überschneidungen geben. Daher empfiehlt es sich, mehrere SROIs zu definieren:

- Der SROI 1 beschreibt die gesellschaftliche Wirkung des Sozialunternehmens in Form von empfangenen und geleisteten Zahlungen an die verschiedenen öffentlichen Hände und Sozialversicherungsträger. Hier werden direkte Zahlungen an das Sozialunternehmen wie Zuschüsse, Leistungsentgelte und direkte Zahlungen des Sozialunternehmens, beispielsweise Unternehmenssteuern und Unternehmensabgaben, Abgaben und Steuern der Beschäftigten, die nicht der Zielgruppe angehören, erfasst. Der SROI 1 gibt damit eine Antwort, ob das Sozialunternehmen an sich - ohne Berücksichtigung der Zielgruppe - Nettozahler oder Nettoempfänger von Transferleistungen der öffentlichen Hand ist.

- Der SROI 2 erfasst die Wirkung des Sozialunternehmens beim Leistungsempfänger. Der SROI 2 beschränkt sich dabei ebenfalls auf Zahlungsströme; die Wirkung wird hier als Transfers von und an die öffentliche Hand definiert. Hier werden also beispielsweise finanzielle Leistungen an die Zielgruppe, Arbeits- und Mobilitätshilfen, Fördermaßnahmen etc. als Input erfasst. Als Output werden dann u. a. Steuern und Abgaben der Zielgruppe herangezogen. In den meis- ten Fällen wird der SROI 2 negativ sein. Der SROI 2 gibt damit eine Antwort, zu welchem Grad den Zahlungsströmen an die Zielgruppe eine Rückzahlung an die öffentliche Hand entgegen steht.

- Mit dem SROI 3 wird dann eine Bewertung der Leistungen des Sozialunternehmens vor dem Hintergrund von vermiedenen Kosten und entgangenen Erträgen aus alternativen Leistungsformen eingeführt. Weiterhin werden hier die indirekten Wirkungen im Umfeld, also beispielsweise bei Angehörigen oder im Sozialraum, soweit wie möglich erfasst. Der SROI 3 ist damit eine Schlüsselkennziffer, die die soziale Vorteilhaftigkeit der Leistungen eines Sozialunternehmens vor dem Hintergrund des Sozialstaats bewerten hilft. Allerdings ist der SROI 3 aufgrund der erforderlichen Kenntnisse über die Wirkung der Leistungen und die Auswirkungen auf das Umfeld durchaus aufwendig zu ermitteln.

- Der SROI 4 legt die Perspektive des Sozialunternehmens auf die regionalökonomische Wirkung eines Sozialunternehmens, also welche Beschäftigung und welche Kaufkraft durch ein Sozialunternehmen in einem Wirtschaftsraum erzeugt werden. Er trifft damit eine Aussage darüber, ob die Ansiedlung eines Sozialunternehmens in einem Wirtschaftsraum vorteilhaft ist.

- Mit dem SROI 5 werden nichtmonetäre Wirkungen erfasst. Hier werden Indikatoren für die Wirkung ermittelt, also beispielsweise Integrationsindikatoren, Lebensqualitätsindikatoren, die dann dem monetären Input gegenübergestellt werden können. Die Ermittlung eines SROI 5 ist eine Entwicklungsaufgabe, für die es einer umfangreichen gemeinsamen Arbeit verschiedener Disziplinen bedarf. Insofern dürfte dieser SROI 5 derzeit eher als Merkposten für künftige Entwicklungen zu sehen sein. 
geführt, etwa für öffentliche Verkehrsmittel, Flughafen- und Messebauten. Eine spezifische Weiterentwicklung von KostenNutzen-Analysen ist der Social Return on Investment (SROI). Der Social Return on Investment hat zum Ziel, die sozialen Wirkungen einer Investition möglichst in Geldgrößen zu bewerten und in Form einer Kennzahl, eben dem Social Return on Investment, auszuweisen.

In den USA wurde der Social Return on Investment ursprünglich entwickelt, um die soziale Wirkung privatwirtschaftlicher Investitionen zu bewerten. In der europäischen Weiterentwicklung steht die Bewertung von sozialen Maßnahmen oder von Sozialunternehmen im Mittelpunkt. An sich sollte der SROI dann eher "SROSI ", also Social Return on Social Investment genannt werden, doch wollen wir der Einfachheit halber bei SROI für Sozialunternehmen bleiben (vgl. Kasten »Fünf SROIs für verschiedene Abnehmersysteme«).

\section{Wertschöpfungskonfigurationen als Lösung für das Steuerungsproblem}

Wenn die Bewertung der Wertschöpfung von Sozialunternehmen mit Hilfe des Social Return on Investment gelingen kann, so bleibt die Frage nach der Steuerung der Wertschöpfung. Wertschöpfung geschieht nicht zufällig oder durch guten Willen allein, sondern mit zielgerichteten Aktivitäten der Leistungserstellung, der »Produktion « von Dienstleistungen. Da sich die Betriebswirtschaft in der Vergangenheit stark auf Produktionsbetriebe konzentriert hatte, fielen die Beschreibungen für die Produktion von Gütern aus betriebswirtschaft- licher Sicht und die Erstellung von sozialen Dienstleistungen aus sozialarbeiterischer Sicht weit auseinander. Diese Lücke führte dazu, dass beide Seiten wenig Verständnis für die jeweils andere aufbrachten. Aus betriebswirtschaftlicher Sicht blieb der Erstellungsprozess Sozialer Arbeit eine große Un- identischer Weise durchlaufen werden kann. Hieraus konnten auch Wertschöpfungspotenziale wie Lerneffekte, Verbesserung von Schnittstellen und Prozessabläufen, Arbeitsteilung durch Konzentration auf Kernkompetenzen und andere analysiert werden.

\section{"Neuere Modelle sehen Dienstleistungen vor allem als Prozesse zur Problemlösung"}

bekannte, die - weil nicht verständlich und beschreibbar - letztlich nur unter Kostenkategorien betrachtet wurde.

Neuere Konzepte der DienstleistungsBetriebswirtschaftslehre ermöglichen es, diese Lücke zu schließen und die Erstellung sozialer Dienstleistungen in das Wertschöpfungskonzept einzubetten. Hier ist allerdings durchaus noch weitere Entwicklungsarbeit zu leisten.

Mitte der 1980-Jahre wurde das Konzept der Wertkette zur Beschreibung der wertschöpfenden Prozesse eines Unternehmens herangezogen. Die Wertkette beschreibt den Wertschöpfungsprozess als eine Abfolge primärer Aktivitäten, die wertschöpfend wirken. Hinzu kommen unterstützende Aktivitäten. Charakteristisch für die Wertkette ist die sequenzielle Abfolge der Aktivitäten und dass der Wertschöpfungsprozess in aller Regel mehrfach und in
Die Wertkette kann - mit erforderlichen inhaltlichen und sprachlichen Anpassungen - als ein Modell zur Darstellung der Wertschöpfung von stark repetitiven sozialen Dienstleistungen beschrieben werden, die aber nur manche Bereiche der Sozialwirtschaft beschreiben (etwa dort, wo rund um den Tagesablauf geplant wird).

Das neuere Modell der Wertshops geht von der Grundannahme aus, dass Dienstleistungen vor allem Problemlösungsprozesse sind. Die Organisation der Wertschöpfung geschieht rund um den Kunden oder genauer: rund um seine Problemlösung. Die gemeinsame Analyse und gemeinsame Arbeit an der Problemlösung sind zentrale Faktoren, ebenso wie die individuelle Steuerung des unternehmerischen Leistungssystems auf den Kunden hin. Wertschöpfungspotenziale bestehen hier insbesondere in der Qualität der Analyse, im Wissensmanage-

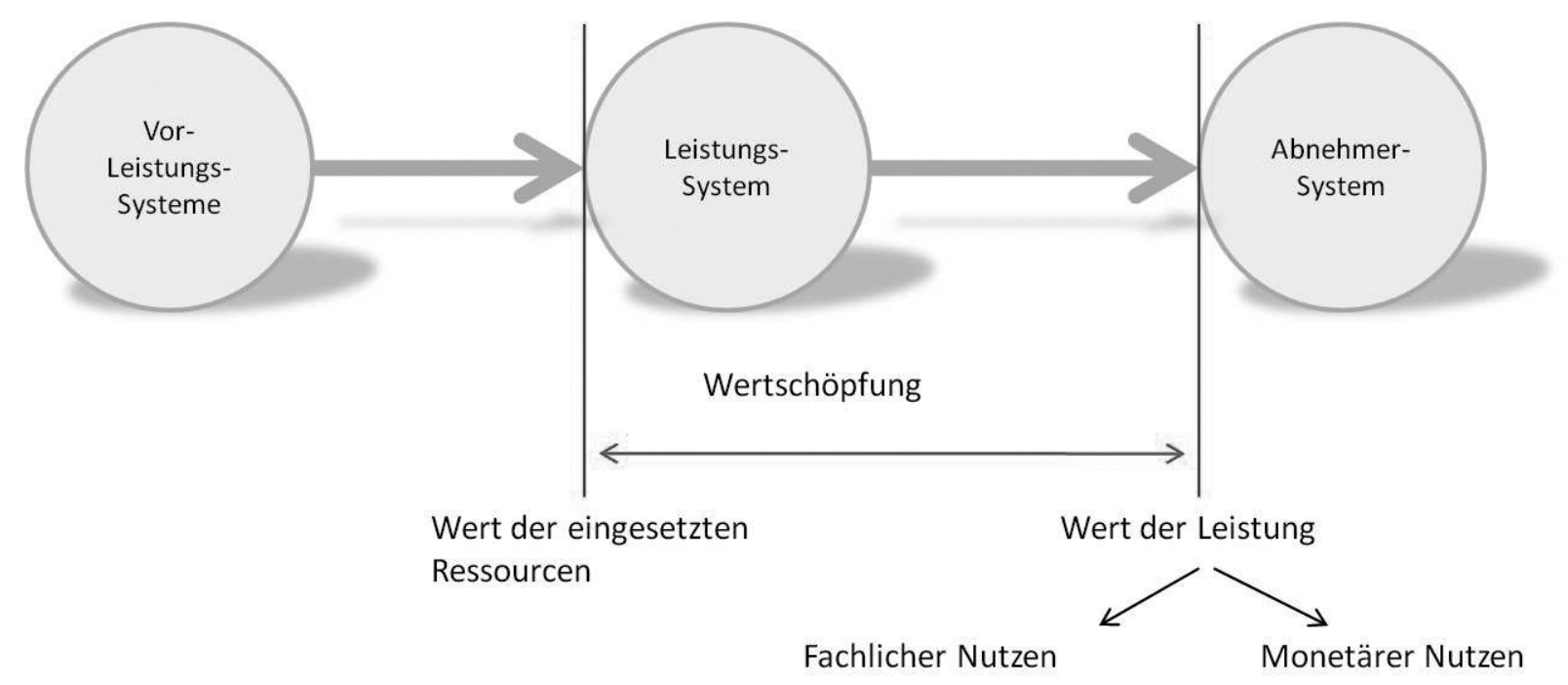




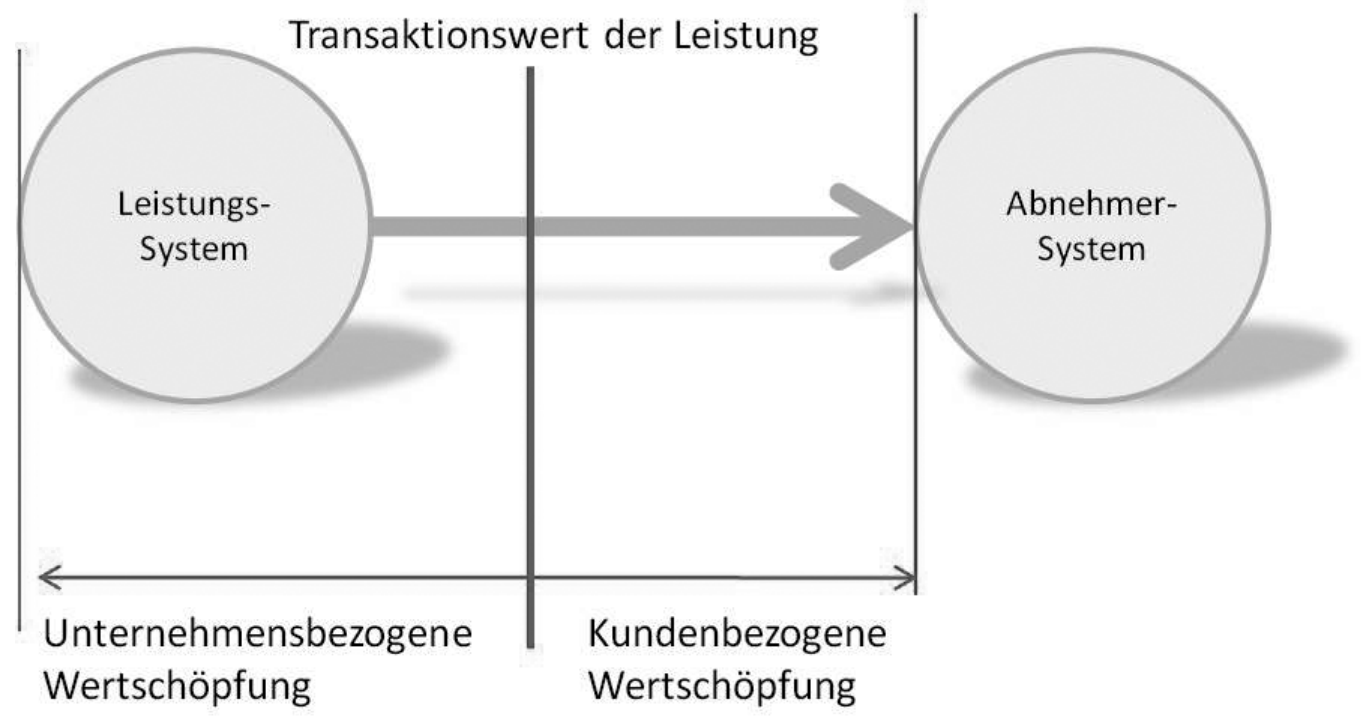

Abb. 2: Der Wert, für den Güter und Leistungen getauscht werden, bestimmt den Transaktionswert und damit die Verteilung der Wertschöpfung zwischen Unternehmen und Kunden.

ment, in den Steuerungsinstrumenten des Leistungssystems (Case Management) und in der Qualität der Problemlösung.

Das Modell des Wertshops kann in vielen Bereichen die fallbezogene Arbeit in Sozialunternehmen beschreiben, wobei vermutlich noch inhaltliche Konkretisierungen und Differenzierungen notwendig sein dürften.

Das Konzept des Wertnetzes beschreibt Wertschöpfung nicht mehr als Transformationsprozess wie die Wertkette oder Problemlösungsprozess wie der Wertshop, sondern als vermittelten Prozess. Der soziale Dienstleister ist intermediär für Angebote, die von anderen erbracht werden. Der Wertschöpfungsprozess besteht in der Schaffung eines Netzwerkes und der tatsächlichen Kontaktvermittlung zwischen Austauschpartnern. Potenziale bestehen hier insbesondere im Know-how und der Fähigkeit zur Schaffung einer Netzwerkinfrastruktur (ggf. auch technologisch) sowie in der Fähigkeit, Vermittlungs- dienste zu betreiben. Diese Wertschöpfungskonfiguration kann dazu dienen, fallübergreifende Arbeit und gemeinwesenorientierte Arbeit zu beschreiben.

\section{Wertschöpfung - der Mehrwert einer gemeinsamen Sprache}

Der besondere Wert der Diskussion um die Wertschöpfung von Sozialunternehmen besteht in der Schaffung einer gemeinsamen Sprache. Während bisher immer wieder Begriffe wie »Ökonomisierung « oder » unproduktive Zeiten « eher Konflikte zwischen den verschiedenen Funktionen und Berufsgruppen in der Sozialwirtschaft betonen, so kann die Betrachtung unter Wertschöpfungskategorien eine gemeinsame Sprache und ein gemeinsames Verständnis des Sozialunternehmens und der sozialen Dienstleistungen herbeiführen. Möglicherweise gelingt es dann, mit weniger Reibungsverlusten »Wertschöpfung « im Sinne der Zielgruppe zu erhöhen.

\begin{tabular}{|l|r|}
\hline Wirtschaftsbereich & In Milliarden Euro \\
\hline Land- und Forstwirtschaft, Fischerei & 20,25 \\
Produzierendes Gewerbe ohne Baugewerbe & 572,72 \\
Verarbeitendes Gewerbe & 517,45 \\
Baugewerbe & 95,23 \\
Handel, Gastgewerbe und Verkehr & 397,43 \\
Finanzierung, Vermietung und Unternehmensdienstl. & 659,16 \\
Öffentliche und private Dienstleister & 494,45 \\
Insgesamt & $2.239,24$
\end{tabular}

Abb. 3: Für das Jahr 2008 listet das Statistische Bundesamt die Werte für die Bruttowertschöpfung einzelner Wirtschaftsbereiche auf (in jeweiligen Preisen, Stand 14.12.2009).

\section{Partnerschaft von Sozialdiensten mit Bürgern.}

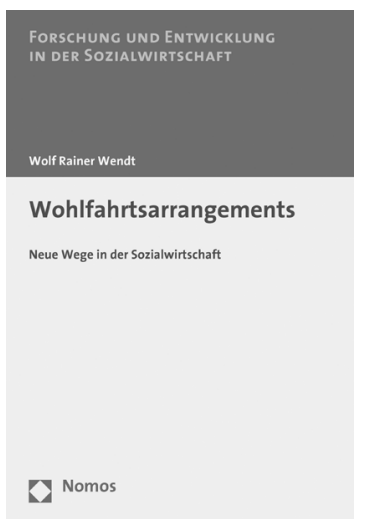

Wohlfahrtsarrangements Neue Wege in der Sozialwirtschaft

Von Prof. Dr. Wolf Rainer Wendt 2010, 132 S., brosch., 24,- $€$, ISBN 978-3-8329-5244-O (Forschung und Entwicklung in der Sozialwirtschaft, Bd. 6) 